\title{
NEW SELF-DUAL CODES OVER GF $(q)$
}

\section{SELESTIN NDJEYA}

Department of Mathematics of Higher Teacher Training College

University of Yaounde I

Cameroon

e-mail: ndjeyas@yahoo.fr

\begin{abstract}
The purpose of this paper is to studies codes $C$ over $G F\left(q^{2 m}\right)$ (where $q$ is a prime power) which are self-dual under a particular product. Using those product we obtained that, all such codes satisfy $C^{\perp}=\bar{C}$ and we determined the number of such codes $C$. Also we have given necessary and sufficient conditions for $C$ to be extended cyclic, and a generator matrix for $C$ on the form $[I \mid A]$,

where $A^{\perp}=\bar{A}$. Finally after enumerated all circulant matrices $A$ with the property $A^{\perp}=\bar{A}$, we also generalize some of the results obtained by Mac Williams, Odlyzko, Sloane and Ward.
\end{abstract}

\section{Introduction}

Self-dual codes are important for a number of practical and theoretical reasons. These codes are of the greatest interest when the weight of the codewords are divisible by a constant. There are several manners to construct codes [1] and self-dual codes over $G F(q)$. One of 
those manners which is due to Wolfmann in [11], who observes that : if $C$ is a $\left[n, \frac{n}{2}\right]$ ( $n$ even) linear code over $G F\left(q^{m}\right)$ which is self-dual under the scalar product $\underline{u} \cdot \underline{v}=\sum_{i=0}^{n-1} u_{i} \cdot v_{i}^{q^{t}}$, where $t$ is a positive integer $0 \leq t \leq m-1$ and if $B=b_{0}, b_{1}, \cdots, b_{m-1}$ is a $G F(q)$-basis of $G F\left(q^{m}\right)$ such that $\operatorname{tr}\left(b_{i} b_{j}^{q^{t}}\right)=\delta_{i j}$, where tr denotes the trace of $G F\left(q^{m}\right)$ over $G F(q)$ [5]. Then the $q$-ary image of $C$ with respect to $B$ is a self-dual $\left(n m, \frac{n m}{2}\right)$ code over $G F(q)[6]$.

In this paper, we are interested in the construction of linear code over $F$ which are self-dual under the scalar product $\underline{u . \bar{v}}=\sum_{i=0}^{n-1} u_{i} v_{i}^{q}(2)$.

So the work is organized as follows. In Section 2, we give the terminology, the symbols and the basic properties of linear codes over $F$ which are self-dual under the scalar product define by (2). In Section 3, we introduce the definition of feasible integers over $F$ and give necessary and sufficient conditions for the extended code of cyclic code over $F$ to be self-dual under the scalar product define by (2). In Section 4, we show that circulant codes $C[2 n, n]$ which are self-dual under the scalar product define by (2) always exist and we enumerate them.

\section{Terminology, Symbols and Basic Properties of Linear Code Over $F$ which are Self-Dual Under the Scalar Product}

We take $F=G F\left(q^{2}\right)$, where $q$ is a power of a prime $p$. A linear code $C$ over $F$ of length $n$ and dimension $k$ consists of $q^{2 k}$ vectors $\underline{u}=\left(u_{0}\right.$, $\left.\cdots, u_{n-1}\right), u_{i} \in F$. 
The weight of $\underline{u}$, denoted by $w t(\underline{u})$ is the number of nonzero $u_{i}$. The minimum distance of $C$ is $d=\min \{w t(\underline{u}): \underline{u} \neq \underline{0} \in C\} . C$ is called a $(n, k, d)$ code.

The dot product is define by (1) $\underline{u \cdot v}=\sum_{i=0}^{n-1} u_{i} \cdot v_{i}$ with the sum evaluated in $F$.

An overbar denotes conjugation, that is, if $x \in F$, then $\bar{x}=x^{q}$. A similar notation is use for vectors, codes. Thus $\bar{u}=\left(u_{0}^{q}, \cdots, u_{n-1}^{q}\right)$ and $\bar{C}=\{\underline{\bar{u}}, \underline{u} \in C\}$. If $C$ is a linear code of length $n$ over $F$; with typical codeword $\left(u_{0}, \cdots, u_{n-1}\right)$, the extended code $C$ consist of the codewords $\left(u_{0}, \cdots, u_{n-1}, u_{\infty}\right)$, with $u_{\infty}=-\sum_{i=0}^{n-1} u_{i}$.

All codes in this paper are linear over $F$ unless stated otherwise.

Let $A_{i}$ be the number of codewords of weight $i$ in $C$.

Then, $W_{c}(x, y)=\sum_{i=0}^{n-1} A_{i} x^{n-1} y^{i}$ is the weight enumerator of $C . C$ is formally self-dual (f.s.d.) if $C$ and $C^{\perp}$ have the same weight enumerator. $C$ is weakly self-dual if $C \subset C^{\perp}$ and self-dual if $C=C^{\perp}$.

$1_{n}$ is the vector $\underbrace{(1,1, \cdots, 1)}_{n \text {-times }}, I$ is the identity matrix and $A^{t}$ denotes the transpose of the matrix $A$.

We have the following theorems and results.

Theorem 2.1. Let $C$ be a linear code over $F$. The following statement are equivalent:

(i) $\bar{C} \subset C^{\perp}$. 
(ii) $\underline{u} \underline{\bar{v}}=0$ for all $\underline{u}, \underline{v} \in C$.

(iii) $\underline{u} \cdot \bar{u}=0$ for all $\underline{u} \in C$.

Proof. We can easily obtain (i) $\Leftrightarrow$ (ii) and (ii) $\Rightarrow$ (iii).

To show (iii) $\Rightarrow$ (ii)

Suppose $\underline{u}, \underline{v} \in C$ and $\alpha$ a primitive element of $F$, then $\underline{u}+\underline{v} \in C$ and $\alpha \underline{u}+\underline{v} \in C$. Then $(\underline{u}+\underline{v})(\underline{\bar{u}}+\underline{\bar{v}})=0 \Rightarrow \underline{u \cdot \bar{v}}=-\underline{v \cdot \bar{u}}$ and $(\underline{\alpha u}+\underline{v}(\alpha \underline{\bar{u}}$ $+\underline{\bar{v}}))=0 \Rightarrow \alpha \underline{u} \cdot \bar{v}=\alpha^{q} \underline{\bar{u}} \cdot \underline{\bar{v}}$. These implies $\left(\alpha-\alpha^{q}\right) \underline{\bar{u}} \cdot \underline{v}=0$ but $\alpha \neq \alpha^{q}$ therefore $\underline{u} \cdot \bar{v}=0$.

Corollary 2.2. If $\bar{C} \subset C^{\perp}$, then $2 k \leq n$.

From Theorem 2.1, we obtain that: if $\bar{C} \subset C^{\perp}$ and $2 k=n$, then $\bar{C}=C^{\perp}$ and $C$ is formally self-dual.

We can also obtain the following result: if $C$ is $(2 m, m)$, code with generator matrix $(I \mid A)$ then, the following statements are equivalent:

(i) $\bar{C}=C^{\perp}$.

(ii) $A \cdot \bar{A}^{t}=-I$.

\section{Extended Cyclic Codes}

In this section, we study cyclic codes $C$ over $F$ for which $C^{e}$ is such that $C^{e \perp}=\bar{C}^{e}$ and we give some examples.

First we recall some important properties:

(i) Let $s$ be a positive integer, the cyclotomic coset of $s \bmod n$ is the set

$C_{s}^{q^{2}}=\left\{s, s q^{2}, \cdots, s q^{2\left(k_{s}-1\right)}\right\}$, where $k_{s}$ is the less integer $k$ such that $q^{2 k} \equiv s(\bmod n)$. 
(ii) Quadratic residue codes are an important subclass of cyclic codes. Let $n$ be an odd prime and let $R$ and $N$ be respectively, the quadratic residues and non-residues of $n$. Then $R$ is a union of cyclotomic cosets $C_{s}^{q^{2}} \bmod n$. Assume that $q^{2} \in R$, the quadratic residue code $Q_{n}$ is the $\left[n, \frac{n-1}{2}\right]$ cyclic code with generator polynomial $\prod_{i \in R}\left(x-\beta^{i}\right)$, where $\beta$ is a primitive $n$-th root of unity of $G F\left(q^{2 k_{1}}\right)$.

Definition 3.1. Let $n$ be an odd positive integer such that $\operatorname{gcd}(n, p)=1, n$ is called a feasible over $F$ if the set $\Omega=1,2, \cdots, n-1$ can be partitioned into disjoint set $I$ and $J$ such that :

(i) $q I=-J(\bmod n)$,

(ii) $I$ (and therefore $J$ ) is and union of cyclotomic cosets $\bmod n$. Otherwise $n$ is infeasible.

We recall the following lemma:

Lemma $3.2([6])$.

(i) $n$ is feasible over $F$ if and only if $C_{s}^{q^{2}} \neq-q C_{s}^{q^{2}}$ for all $s$ with $1 \leq s$ $\leq n-1$.

(ii) $n$ be an odd positive integer such that $\operatorname{gcd}(n, q s)=1$ for all integers $s<n$. If for all integer $i<n, q^{2 i-1}+1$ and $n$ are relatively prime, then $n$ is feasible over $F$.

(iii) Let $n$ be an odd prime, consider e the multiplicative order of $q$ in $\frac{\mathbb{Z}}{n \mathbb{Z}} \backslash\{0\}$. If $e \equiv 0(\bmod 4)$ or $e \equiv 1(\bmod 2)$, then $n$ is feasible over $F$.

(iv) Let $n=4 k q-1$ be a prime, where $k$ is a positive. If $q$ is a quadratic residue $(\bmod n)$, then $n$ is feasible over $F$.

(v) Let $n=4 k q+l$, where $k$ and $l$ are positive integers with $l \equiv 1(\bmod 4)$ and $\operatorname{gcd}(n, q)=1$. If $q$ is a quadratic non-residue $\bmod n$, then $n$ is feasible. 
We have the following important theorem:

Theorem 3.3. Let $n$ be a feasible integer over $F$ with $n \equiv-1(\bmod p)$.

Let $\beta$ be a primitive $n$-th root of unity in $G F\left(q^{2 k_{1}}\right)$ and $\Omega=I \cup J$ is the partition specified by the Definition 3.1, then the extended code $C^{e}$ of the cyclic code $C$ of length $n$ with zeros $\left\{\beta^{i}, i \in I\right\}$ is such that $C^{e}=\bar{C}^{e \perp}$.

Conversely, if $n$ is an odd integer with $\operatorname{gcd}(n, p)=1$ and $n>p$. Suppose there exists a cyclic code $C$ over $F$ verifying following conditions :

(i) $C$ is an $\left[n, \frac{n+1}{2}\right]$ code over $F$ and the extended code $C^{e}$ of $C$ is a linear code over F such that $C^{e}=\bar{C}^{e \perp}$.

(ii) $1_{n}$ is not a zero of $C$.

Then $n$ is feasible over $F$ and $n \equiv-1(\bmod p)$.

Proof. Let $n$ be a feasible integer over $F$ with $n \equiv-1(\bmod p)$.

Let $C$ be the cyclic code of length $n$ over $F$ with zeros $\left\{\beta^{i}, i \in I\right\}$.

Let $C^{e}$ be the extended code of $C$. Then $C^{e}$ is a linear code $[n+1$, $\left.\frac{n+1}{2}\right]$ over $F$ since $\operatorname{gcd}(n, p)=1$.

Now $\bar{C}^{e}$ is then extension of $\bar{C}$ and $C^{e \perp}$ is generated by $1_{n+1}$ and $\left\{|\underline{u}| 0 \mid: \underline{u} \in C^{\perp}\right\}[6]$.

But $\operatorname{dim}_{F}\left(C^{e \perp}\right)=\operatorname{dim}_{F}\left(\bar{C}^{e}\right)=\frac{n+1}{2}(*)$.

Therefore, we will only show that $\bar{C}^{e} \supset C^{e \perp}$. 
The zero of $C^{\perp}$ are $\left\{\beta^{-j}, j \in J \cup\{0\}\right\}$ and the zero of $\bar{C}$ are $\left\{\beta^{i q}, i \in I\right\}$.

Since $n$ is feasible, we have $q I=J(\bmod n)$.

$$
\Rightarrow \bar{C} \supset C^{\perp}
$$

Since $0 \notin I \Rightarrow I_{n} \in C \cap \bar{C}$

$$
\Rightarrow I_{n+1} \in \bar{C}^{e^{\perp}} \text { since } n \equiv-1(\bmod p) \text {. }
$$

But $\bar{C} \supset C^{\perp}$ and $I_{n} \in C \cap \bar{C}$

$$
\Rightarrow \forall \underline{u} \in C^{\perp},|\underline{u}| 0 \mid \in C^{e} \text {. Therefore } \bar{C}^{e} \supset C^{e \perp} \text { and }\left(^{*}\right) \Rightarrow \bar{C}^{e} \supset C^{e \perp} \text {. }
$$

Conversely, suppose there exists a cyclic code $C$ over $F$ with parameters $\left[n, \frac{n+1}{2}\right]$ verifying the conditions (i) and (ii).

Suppose the zeros of $C$ are $\left\{\beta^{i}, i \in I\right\}$.

- $I \subset \Omega$;

- Put $J=\Omega-I$, then $\bar{C}$ has zeros $\left\{\beta^{i q}, i \in I\right\}$ and $\bar{C}^{\perp}$ has zero $\left\{\beta^{-j}, j \in J \cup\{0\}\right\}$ since $\operatorname{gcd}(n, p)=1 \Rightarrow|I|=\frac{n+1}{2}$,

$$
\begin{aligned}
& 0 \notin I \Rightarrow I_{n} \in C \cap \bar{C} \text { since } \bar{C}^{e} \text { and } I_{n+1} \in \bar{C}^{e} \\
& \Rightarrow n \equiv-1(\bmod p) \text { but } \forall \underline{u} \in \bar{C}^{\perp} ;|\underline{u}| 0 \mid \in \bar{C}^{e} \\
& \Rightarrow \bar{C} \supset C^{\perp} \\
& \Leftrightarrow-J \cup\{0\} \supset q I(\bmod n)
\end{aligned}
$$


$\Rightarrow q J \equiv-J(\bmod n) ;$ and $n$ is feasible over $F$.

Remark 3.4. In the Theorem 3.3, the roles of $I$ and $J$ are symmetric.

Example 3.5. $F=G F\left(3^{2}\right)=G F(3)(\alpha)$, where $\alpha=\alpha+1, n=5$, $\operatorname{gcd}(3,5)=1$.

$$
C_{0}^{0}=\{0\} ; C_{1}^{2}=\{1,4\} ; C_{0}^{0}=\{2,3\}=J .
$$

$I \cap J=\emptyset, \Omega=I \cup J, 3 I \equiv-J(\bmod 5)$. Then 5 is feasible over $F$ and $n \equiv(\bmod 3)$.

Let $C$ be the cyclic code with zeros $\left\{\beta^{1}, \beta^{4}\right\}$, where $\beta=\gamma^{16}$ with $\gamma^{2}=\gamma+\alpha^{5}$. C has generator polynomial $g(x)=x^{2}+\alpha^{2} x+1$ and $C^{e}$ is a [6, 3]-linear code over $F$ such that $\bar{C}^{e}=C^{e \perp}$.

We end this section with some examples of quadratic residues codes such that $\bar{Q}^{e}=Q_{n}^{e \perp}$.

\section{Table}

\begin{tabular}{|c|c|c|c|c|}
\hline$q$ & 3 & 7 & 11 & 19 \\
\hline$n$ & $12 k+5$ or $12 k-1$ & $28 k+13$ or $28 k-1$ & $44 k+21$ or $44 k-1$ & $76 k+37$ or $76 k-1$ \\
\hline
\end{tabular}

\section{Circulant Codes}

In this section, we caracterize circulant codes and we enumerate them.

We denote by $R_{n}$ be the algebra $F(x) /\left(x^{n}-1\right)$ and we recall the following definitions and results.

Definition 4.1. An $n \times n$ circulant matrix over $F$ is a matrix of the form: 


$$
a=\left[\begin{array}{cccc}
a_{0} & a_{1} & \cdots & a_{n-1} \\
a_{n-1} & a_{0} & \cdots & a_{n-2} \\
\cdots & \cdots & \cdots & \cdots \\
a_{1} & a_{2} & \cdots & a_{0}
\end{array}\right],
$$

where $a_{i} \in F$ for all $i=0, \cdots, n-1$.

Definition 4.2 ([8]). A linear code $C$ is said to be circulant if it has a generator matrix of the form $G=(I \mid A)$, where $A$ is an $n \times n$-circulant matrix.

Proposition 4.3 ([8]). The algebra of $n \times n$-circulant matrices is isomorphic to the algebra $R_{n}$. The isomorphism is given by: $A \mapsto \underline{a}(x)=$ $\sum_{i=0}^{n-1} a_{i} x^{i}$

Proposition 4.4 ([8]). Let $\tau$ be the application of $R_{n}$ defined by $\tau(\underline{a}(x))=\underline{a}\left(\overline{x^{-1}}\right)$. Then $\tau$ is an involutive $G F(q)$-automorphism.

Lemma 4.5 ([8]). Let $n$ be a positive integer with $\operatorname{gcd}(n, p)=1$.

(1) If $C_{n}$ is the set of all linear circulant codes $C[2 n ; n]$ over $F$ such that $\bar{C}=C^{\perp}$, then $\left|C_{n}\right|>0$.

(2) If $C(n)$ is the set of all representants of cyclotomic cosets modulo $n$ over F, then:

(i) $R_{n}=\bigoplus_{s \in C(n)} M_{s}$, where $M_{s}$ is a minimal ideal generated primitive idempotent $\theta_{s}(x)$.

(ii) $\sum_{s \in C(n)} \theta_{s}(x)=1$ and $\theta_{i}(x) \theta_{j}(x)=\delta^{i j} \theta_{i}(x)$ for all $i$ and $j \in C(n)$, where $\delta_{i j}$ is the Kronecker symbol. 
(iii) If $\operatorname{dim} M_{s}=m_{s}$, then $M_{s}$ is isomorphic to $G F\left(q^{2 m_{s}}\right)$. One such isomorphism is given by, $\phi_{\beta}: \underline{a}_{s}(x) \in M_{s} \mapsto \underline{a}_{s}(\beta)=\phi_{\beta}\left(\underline{a}_{s}(x)\right)$, where $\beta$ primitive $n$-th root of unity such that $\theta_{s}(\beta)=1 ; \beta \in G F\left(q^{2 m_{s}}\right) ; \phi_{\beta}\left(-\theta_{s}\right.$ $(x))=-1$ and if $\bar{M}_{s}^{t}=M_{s}$, then for all $\underline{a}_{s}(x) \in M_{s}, \phi_{\beta} \circ \tau\left(\underline{a}_{s}(x)\right)=$ $\underline{a}_{s}(\beta)^{q m_{s}}$.

Lemma 4.6 ([8]). Let $\underline{a}(x) \in R_{n}$ and $\underline{b}(x) \in M_{t}$ such that $\underline{a}(x) \underline{b}(x)=$ $\theta_{t}(x)$. Put $\underline{c}(x)=\underline{a}(x)+\underline{b}(x)$ and $m_{t}=\operatorname{dim}_{F} M_{t}$ then, there are $q^{2 m_{t}}-1$ ways of choosing $\underline{c}(x)$.

Lemma 4.7 ([10]). Let $(G ; x)$ be a cyclic group of order $n$. Then in $G$, the equation $x^{n}=a$ has $d$ solutions if $a^{\delta}=1$ and zero solution if $a^{\delta} \neq 1$, where $d=\operatorname{gcd}(n, r)$ and $\delta=\frac{n}{d}$.

Remark 4.8. For $n$ odd, set: $x^{n}-1=(x-1) \prod_{i} g_{i}(x)$ and for $n$ even, set : $x^{n}-1=(x-1)(x+1) \prod_{j} f_{j}(x)$, where $g_{i}(x)$ and $f_{i}(x)$ are distinct linear factors.

We have the following important theorem show how to construct circulant codes $C$ such that $\bar{C}=C^{\perp}$ and enumerate them.

Theorem 4.9. (a) Let $n$ be a positive integer with $\operatorname{gcd}(n, p)=1$. Let

$$
\begin{aligned}
F=G F\left(q^{2}\right), R_{n}= & \frac{F(x)}{x^{n}-1} . \\
& S_{1}=\left\{s \in \mathbb{N}, s<n \mid C_{s}^{q^{2}}=-C_{-q s}^{q^{2}}\right\},
\end{aligned}
$$

and

$$
S_{2}=\left\{(s, t) \in \mathbb{N}^{2},(s \neq t), s<n \text { and } t<n \mid C_{s}^{q^{2}}=-C_{-q t}^{q^{2}}\right\}
$$


If $C_{n}$ is the set of all linear circulant codes $C(2 n, n)$ over $F$ such that $\bar{C}=C^{\perp}$, then we have the following properties :

(i) If $S_{2}=\emptyset$, then $\left|C_{n}\right|=(q+1)^{n}$.

(ii) If $S_{2} \neq \emptyset$, then $\left|C_{n}\right|=\epsilon \prod_{s}\left(q^{k(s)}+1\right) \prod_{t}\left(q^{k(t)}-1\right)$,

where $\epsilon=\left\{\begin{array}{c}1 \text { if } n \text { is odd, } \\ 2 \text { if } n \text { is even, }\end{array}\right.$ and $\left\{\begin{array}{c}k(s)=\left|C_{s}^{q^{2}}\right| \text { with } s \in S_{1}, \\ k(t)=\left|C_{-q t}^{q^{2}}\right| \text { with }(s, t) \in S_{2},\end{array}\right.$

where $k(s)$ contributes one term to the first product and every pair $(s, t)$ contributes one term to the second product.

(b) If $F=G F\left(3^{2}\right)$ and $n=3 s$, where $s$ is a positive integer, then: $\left|C_{n}\right|=9^{s}\left|C_{s}\right|$.

Proof. A circulant matrix $A$ with first row $\left(a_{0} a_{1} \cdots a_{n-1}\right)$ is specified by the element $\underline{a}(x)=\sum_{i=0}^{n-1} a_{i} x^{i}$ of $R_{n}$. Then $\bar{A}$ and $A^{t}$ are represented by :

$$
a(\bar{x})=\sum_{i=0}^{n-1} a_{i}^{q} x^{i} ; \quad \underline{a}(x)^{t}=\sum_{i=0}^{n-1} a_{i} x^{n-i}
$$

$\tau(\underline{a}(x))=\bar{a}^{t}(x)$ and $A \bar{A}^{t}=-I$ if and only if $\underline{a}(x) \tau(\underline{a}(x))=-1$ in $R_{n}$.

Case (a). $\operatorname{gcd}(n, p)=1$; then $R_{n}$ is a semi simple group algebra over $F$ which is direct sum of its minimal ideals (Lemma 4.5). $R_{n}=\underset{s \in C(n)}{\bigoplus} M_{s}$.

The idea is to range minimal ideals as follows: $M_{i}=\tau\left(M_{i}\right)$ for $0 \leq i \leq 1$ and $M_{i}=\tau\left(M_{i+1}\right)$ for $1 \leq i \leq r$ when $n>2$. The proof of (i) and (ii) is immediate from Lemma 4.7. 
Suppose $\underline{a}(x)=\sum_{s=0}^{1} \underline{a}_{s}(x)+\sum_{t=1+0,1+3, \cdots}\left(C_{t}(x)+C_{t+1}(x)\right)$ with $\underline{a}_{s}(x) \in M_{s}$;

$C_{t}(x) \in M_{t} \quad$ then $\quad \underline{a}(x) \tau(\underline{a}(x))=\sum_{s=0}^{1} \underline{a}_{s}(x) \tau\left(\underline{a}_{s}(x)\right)+\sum_{t=1+1,1+3, \cdots}\left(C_{t}(x) \tau\right.$

$\left.\left(C_{t+1}(x)\right)+\tau\left(C_{t}(x)\right) C_{t+1}(x)\right)$ but $-1=\sum_{s=0}^{1}\left(-\theta_{s}(x)\right)+\sum_{t=1+1,1+3, \cdots}\left(-\theta_{t}(x)\right)$ $\left(-\theta_{t+1}(x)\right)$.

Then we have $\underline{a}(x) \tau(\underline{a}(x))=-1$ in $R_{n}$.

$$
\Leftrightarrow\left\{\begin{array}{l}
\underline{a}_{s}(x) \tau\left(\underline{a}_{s}(x)\right)=\theta_{s}(x)(*) \\
C_{t}(x) \tau\left(C_{t+1}(x)\right)=-\theta_{t}(x)(* *)
\end{array}\right.
$$

If $m_{s}=\operatorname{dim}_{F} M_{s}$ and $m_{t}=\operatorname{dim}_{F} M_{t}$, from the Lemmas 4.5, 4.6, and 4.7 and the relations $(*)$ and $(* *)$, we obtain the results (i) and (ii).

Case (b). $n=3 s$ and $F=G F\left(3^{2}\right) . R_{n}$ is no longer semi simple. Let $I_{s}$ be the ideal of $R_{n}$ generated by $x^{s}-1$ with $I_{s}=\tau\left(I_{s}\right)$. Then $\left|I_{s}\right|=9^{s}$ circulant in $R_{s}$.

Suppose $\underline{a}(x) \bar{a}^{\underline{a}(x)}{ }^{T_{n}}=-1(*)$.

We can also write : $\underline{u}(x)=\underline{a}(x)+\underline{w}(x)$, where $\underline{w}(x) \in I_{s}$

$$
\Rightarrow \underline{a}(x)=\underline{u}(x)-\underline{w}(x) .
$$

Then $\underline{a}(x) \bar{a}^{\underline{a}(x)}{ }^{T_{n}}=-1 \Rightarrow \underline{u}(x) \bar{u}^{\underline{u}(x)}{ }^{T_{s}}=-1\left({ }^{* *}\right)$ and $\underline{u}(x) \neq 0$.

Put $\underline{r}(x)=\bar{u}^{\underline{u}(x)}{ }^{T_{n}} \underline{w}(x)$ then $\underline{r}(x) \in I_{s}$ since $\underline{w}_{s}(x) \in I_{s}$.

$(*) \Rightarrow \underline{u}(x) \bar{u}^{(x)}{ }^{T_{n}}=-1+\underline{r}(x)+\underline{r}{ }^{T_{n}}{ }^{(* * *)}$; we then obtain: 


$$
\underline{a}(x)=\underline{a}(x)+\frac{2 \underline{r}(x)}{\overline{\bar{u}(x)} T_{s}}+\frac{\underline{r}(x) \underline{r(x)} T_{s}}{\underline{u}(x)(\underline{\bar{u}(x)} T)^{2}}(* * * *)
$$

Conversely, given $\underline{u}(x) \in R_{s}$ satisfying the relation $(* *)$

There are $9^{s}$ of $\underline{r}(x) \in I_{s}$ satisfying $(* * *)$

And therefore $9^{s} \underline{a}(x)$ given by $(* * *)$

Hence $\left|C_{n}\right|=9^{s}\left|C_{s}\right|$.

Example 4.10. $F=G F(9)=G F(3)(\alpha)$, where $\alpha^{2}=\alpha+1 . N=3$ then $s=1$. $R_{1}=F . u \in R_{1}$, with $u^{4}=-1 \Leftrightarrow u \in\left\{\alpha, \alpha^{3}, \alpha^{5}, \alpha^{7}\right\}$. Take $u=\alpha \quad$ and $\quad \underline{r}(x)=\alpha^{7} x^{2}+\alpha x+\alpha^{2}$, then $\underline{a}(x)=\alpha^{3} x^{2}+x+\alpha^{3} \quad$ is circulant and $\left|C_{3}\right|=9\left|C_{1}\right|=9 \times 4=36$.

Remark 4.11. The result obtained in this paper generalized the work in [2], [3], [4], [6], [7], [9].

\section{Conflict of Interest}

The author declare that there is no conflict of interest regarding the publication of this paper.

\section{Acknowledgement}

I thank J. Wolfmann and all the members of G.R.I.M. of Toulon for their constructive remarks. Further, I would also like to thank the anonymous referee for valuable comments on the submitted manuscript.

\section{References}

[1] T. S. Atamewoue, Y. B. Jun, C. Lele, S. Ndjeya and S. Z. Song, Codes bases on residuated lattices, Commun. Korean Math. Soc. 31(1) (2016), 27-40.

[2] J. Kim and Y. Lee, Euclidean and Hermitian self-dual MDS codes over large finite fields, Journal of Combinatorial Theory, Series A 105 (2004), 79-95. 
[3] F. J. Mac Williams, A. M. Odlyzko and N. J. A. Sloane, Self-dual codes over GF(4), Journal of Combinatorial Theory, Series A25 (1978).

[4] F. J. Mac Williams and N. J. A. Sloane, Orthogonal circulant matrices over finite fields and how to find them, Journal of Combinatorial Theory 10 (1971).

[5] F. J. Mac Williams and N. J. A. Sloane, The Theory of Error Correcting Codes, North Holland Mathematical Company, 1983.

[6] S. Ndjeya, Hermitian Codes, Ph.D Thesis, University of Marseille II, France, 1988.

[7] S. Ndjeya, On the Hermitian trace basis of $G F\left(q^{2 m}\right)$ over $G F(q)$, Journal of Algebra Number Theory: Advances and Applications 17(2) (2017), 85-92.

Available at http://scientificadvances.co.in DOI: http://dx.doi.org/10.18642/jantaa7100121833.

[8] A. Poli and C. Rigoni, Enumeration of self-dual $2 k$ circulant code, Lecture Notes in Computer Science. AAECC-2, Toulouse, France, 1984.

[9] P. Rabizzoni, On $q$-ary Image Codes over an Extension of $G F(q)$, Ph.D Thesis, Toulon and Var University, France, 1982.

[10] A. Warusfel, Finite Algebraic Structures, Hachette University, Paris, France, 1971.

[11] J. Wolfmann, Different aspects of the $q$-ary image codes, Treatment of the Signal, France 1(2) (1984). 